\title{
Vincenzo Foppa nella Cappella Portinari in Sant'Eustorgio: dall'exemplum nella predicazione e nella letteratura domenicana alla narrazione per immagini
}

Vincenzo Foppa dans la chapelle Portinari, à Sant'Eustorgio : de l'exemplum dans la prédication et la littérature dominicaine au récit par images Vincenzo Foppa in Portinari Chapel in Saint Eustorgio: From the Exemplum in the Preaching and in Dominican Literature to a Narrative by Images

\section{Rita Capurro}

\section{(2) OpenEdition}

Edizione digitale

URL: http://journals.openedition.org/cei/5997

DOI: $10.4000 /$ cei.5997

ISSN: 2260-779X

Editore

UGA Éditions/Université Grenoble Alpes

Edizione cartacea

ISBN: 978-2-37747-159-1

ISSN: 1770-9571

Notizia bibliografica digitale

Rita Capurro, «Vincenzo Foppa nella Cappella Portinari in Sant'Eustorgio: dall'exemplum nella predicazione e nella letteratura domenicana alla narrazione per immagini», Cahiers d'études italiennes [Online], 29 | 2019, online dal 30 septembre 2019, consultato il 26 mars 2021. URL: http:// journals.openedition.org/cei/5997 ; DOI: https://doi.org/10.4000/cei.5997

Questo documento è stato generato automaticamente il 26 mars 2021. 


\title{
Vincenzo Foppa nella Cappella Portinari in Sant'Eustorgio: dall'exemplum nella predicazione e nella letteratura domenicana alla narrazione per immagini
}

\author{
Vincenzo Foppa dans la chapelle Portinari, à Sant'Eustorgio : de l'exemplum \\ dans la prédication et la littérature dominicaine au récit par images \\ Vincenzo Foppa in Portinari Chapel in Saint Eustorgio: From the Exemplum in \\ the Preaching and in Dominican Literature to a Narrative by Images
}

Rita Capurro

1 Nel progetto complessivo della Cappella Portinari in Sant'Eustorgio a Milano, emergono due elementi fusi mirabilmente: da un lato l'aspetto stilistico di novità per Milano con un'architettura che richiama la sacrestia vecchia di San Lorenzo a Firenze, rispondente alla sensibilità fiorentina del committente Pigello Portinari; dall'altro, gli affreschi di Vincenzo Foppa, in attenta relazione con gli spazi, rispondono con figurazioni intrise di naturalismo alla tradizione dell'iconografia domenicana, narrativa e didascalica, ideale per rafforzare l'impianto di sollecitazione multisensoriale nel corso delle predicazioni. Nel presente contributo si propone una rilettura del capolavoro dell'arte lombarda del $\mathrm{XV}$ secolo, alla luce di alcune considerazioni multidisciplinari (di storia, storia della Chiesa, storia degli Ordini religiosi), e sugli effetti generati dalla compresenza di diversi attori con esigenze, linguaggi, obiettivi differenti. In particolare, il ciclo foppesco è analizzato alla luce degli exempla di vite di santi che caratterizzano parte della predicazione e letteratura domenicana del XV secolo e che vedono tra i protagonisti san Pietro da Verona martire, notevole modello per i domenicani lombardi, alle prese in quegli anni con un complesso processo di riforma. 


\section{Il contesto}

Nel 1464, quando iniziarono i lavori per la realizzazione della Cappella Portinari, la basilica di Sant'Eustorgio aveva già dieci secoli di vita e dall'insediamento dei padri domenicani avvenuto nel 1220, aveva assunto crescente prestigio come sede del tribunale dell'Inquisizione dal 1234. In seguito Sant'Eustorgio si distinse come convento dove aveva operato, fino al momento del suo martirio nel 1252, Pietro da Verona ${ }^{1}$, il secondo santo domenicano dopo il fondatore Domenico ${ }^{2}$. Inoltre, il convento divenne un luogo di formazione teologica dal 1262 con la presenza dello studium logicee per la provincia di Milano e, dal 1315, dello studium philosophice moralis ${ }^{3}$.

3 Per meglio comprendere il ruolo dei domenicani di Sant'Eustorgio nel XV secolo, è utile ricordare che, quando tra gli anni Venti e Quaranta del XV secolo l'Ordine attraversò il processo di riforma, diversi padri di Sant'Eustorgio, quale, ad esempio, Pietro d'Alzate per Piacenza, furono protagonisti nel condurre i delicati passaggi. Inoltre, sul piano politico locale, Francesco Sforza ebbe relazioni molto strette con diversi ordini religiosi ma soprattutto con i domenicani ${ }^{4}$. Il domenicano Girolamo Visconti, definito da Bianca Maria "servo suo», venne supportato dai duchi nella carica di provinciale della provincia lombarda dedicata a san Pietro martire: tale incarico gli venne infatti assegnato una prima volta nel 1462-1463, al tempo di Francesco Sforza, poi nel 1471-1472 al tempo di Galeazzo Maria. Il XV secolo vide i domenicani di Sant'Eustorgio protagonisti della predicazione a Milano. I padri non esercitavano la loro missione esclusivamente nell'ambito di Sant'Eustorgio ma erano incaricati già dalla seconda metà del XIII secolo, per decisione del vescovo Ottone Visconti, di tenere lezioni di teologia presso il duomo di Milano ${ }^{6}$. Anche in veste di predicatori la loro prestigiosa presenza si distingueva presso la cattedrale di Milano e il consenso nei loro confronti fu tale che nel 1440, quando il domenicano Alessandro da Bologna fu incaricato per un ciclo di prediche quaresimali in duomo, la Fabbrica prese la risoluzione di spostare un muro per accrescere lo spazio per il pubblico ${ }^{7}$.

Nel 1465 il conte Vimercati, uno dei massimi sostenitori alla scalata al titolo di duca di Visconti, donò il terreno per Santa Maria delle Grazie ${ }^{8}$. Nel 1559, il trasferimento del Tribunale dell'Inquisizione ai Domenicani osservanti di Santa Maria delle Grazie segnò l'inizio del declino per Sant'Eustorgio.

\section{Fondazione della cappella e il ciclo decorativo}

Dopo il martirio, il corpo di san Pietro da Verona trovò collocazione presso la basilica di Sant'Eustorgio, conferendo al luogo nuovo prestigio, grazie alla devozione che immediatamente riscosse il martire per la fede. I resti mortali ebbero una sistemazione provvisoria all'interno della basilica fino al 1340 quando furono traslati nella straordinaria arca realizzata da Giovanni di Balduccio tra 1335 e 1339, su commissione dei Domenicani che per l'occasione fecero una colletta in tutto il mondo'. Solo nel XVIII secolo il monumento sepolcrale con il corpo del santo fu spostato dalla chiesa e collocato nella cappella Portinari ${ }^{10}$ dove si trova tuttora. Al momento della traslazione del corpo del santo nell'arca, l'arcivescovo Giovanni Visconti ne aveva trattenuto il capo, forse per duplicare i luoghi destinati alla devozione ma lo aveva dovuto restituire a Sant'Eustorgio in una preziosa teca per rispondere alle severe proteste popolari. La testa fu inizialmente collocata in luogo separato, presso la sacrestia, quindi si cominciò 
a immaginare la creazione di un luogo preposto alla sua custodia già nel 1441 quando Paolino Brivio, miracolato del santo dispose, in accordo con il suo padre spirituale Pietro d'Alzate, di rifare una cappella per valorizzare la reliquia. Di fatto la traslazione avvenne solo con la realizzazione della cappella Portinari che i documenti antichi citano come "Cappella Sancti Petri martyris ad caput»" ${ }^{11}$. Gaspare Bugati nella sua storia inedita di Sant'Eustorgio fino al $1577^{12}$, scrive che il Portinari «fece fare [...] la cappella del capo di s. Pietro martire per la gran devotione ch'ebbe al santo». Il sepolcro del committente in segno di devota dedicazione è collocato sotto il pavimento della cappella, lo stemma della sua casata nella lanterna e la sua effige rappresentata in un dipinto attribuito a Giovanni da Vaprio, attualmente collocato presso l'altare. La cappella è quindi in primis omaggio al santo martire e solo in seconda istanza e rispettosamente in secondo piano, cappella funeraria per Pigello Portinari.

6 La cappella fu costruita a ridosso della basilica, con accesso esclusivo tramite la sacrestia. L'artefice della costruzione realizzò con la cappella Portinari un'architettura chiaramente ispirata all'archetipo fiorentino brunelleschiano nell'ispirazione alla sacrestia vecchia di San Lorenzo ${ }^{13}$.

7 Il ciclo decorativo della cappella si sviluppa su un progetto che segue armonicamente un'unica regia, e comprende nel tamburo di imposta della cupola una schiera di angeli danzanti in terracotta dipinta, che sono rievocati anche nella decorazione delle paraste, di artista ignoto ma di cultura lombarda, e i dipinti murali di Foppa con il cielo della cupola a scaglie policrome, negli otto oculi chiusi della cupola figure di santi prive di attributi, nei pennacchi i quattro dottori della Chiesa: san Gregorio Magno, san Girolamo, sant'Ambrogio e sant'Agostino. Le scene principali raffigurano sulla parete di fronte all'ingresso l'Annunciazione, sulla parete opposta l'Assunzione della Vergine, sulla parete sinistra il Miracolo del piede risanato e il Martirio di san Pietro Martire, sulla parete destra il Miracolo della nube e il Miracolo della falsa Madonna.

Le pareti della cappella furono coperte da intonaci alla metà del XVII secolo e tornarono alla luce solo nel 1871.

Roberto Longhi a partire dai suoi primi studi sull'arte lombarda nel 1911, ma ancora ribaditi nel 1929 nei Quesiti caravaggeschi, identifica negli affreschi della cappella Portinari quegli elementi di naturalismo lombardo che segnano tutto il percorso specifico dell'arte in Lombardia che vedrà in Caravaggio la sua espressione più alta. Tutta la storiografia artistica successiva ribadisce questo concetto, contribuendo a una definizione dell'arte lombarda nel panorama artistico del Quattrocento ${ }^{14}$.

10 Per la Cappella Portinari, i molti studi si sono concentrati su considerazioni puntuali sulle diverse partiture del complesso decorativo, approfondendo, di volta in volta, questioni relative alla committenza di Pigello Portinari, influente banchiere del Banco Mediceo a Firenze, che in qualche modo si fa da tramite per fare tradurre anche da artisti locali le nuove istanze rinascimentali dell'arte toscana ${ }^{15}$, oppure sulla personalità artistica di Vincenzo Foppa, straordinario interprete della pittura del XV secolo in Lombardia nell'unico ciclo pittorico che è giunto pressoché integro fino ai nostri giorni. I Domenicani, al tempo titolari della basilica di Sant'Eustorgio, sono generalmente considerati dalla storiografia artistica nella narrazione complessiva delle vicende della cappella, in relazione alla devozione del santo martire domenicano Pietro da Verona Martire che è protagonista di alcuni dipinti del Foppa. Un contributo che invece pone in luce la rilevanza della relazione tra pittura e predicazione domenicana anche per 
sottolineare come questa sia una concausa della ricerca di veridicità delle scene della vita di Pietro martire è di Dalai Emiliani che sottolinea:

Non la responsabilità dell'inventio spettò al Foppa, cioè la «scelta dei pensieri finalizzati» da tradurre nelle historic; e solo in parte forse egli si impegnò nella dispositio cioè nell'organizzazione significante dei materiali iconografici, che sembra piuttosto improntata alle sperimentatissime regole della tradizione retorica domenicana, mentre solo sua fu la ricerca della compositio, delle forme e del linguaggio più efficaci per comunicare visivamente ${ }^{16}$.

11 Partendo da qui, di seguito si tenterà di valorizzare il contributo dei Padri predicatori alla realizzazione del ciclo pittorico, attraverso una breve analisi sulle azioni di promozione per la devozione del santo domenicano, mediante gli strumenti concorrenti di arte, agiografia e predicazione.

\section{Il complesso narrativo delle immagini}

Per una lettura iconografica coerente è necessario considerare il complesso decorativo della cappella nel suo insieme e un confronto con la struttura della composizione narrativa anche del monumento funebre di Giovanni di Balduccio ${ }^{17}$. Infatti, sebbene all'epoca della costruzione della cappella Portinari, l'arca si trovasse nella quinta campata sinistra della basilica di Sant'Eustorgio, si trattava di un riferimento importante sia da un punto di vista iconografico, essendo il più ricco repertorio in Sant'Eustorgio dedicato a san Pietro, sia, e principalmente, da un punto di vista devozionale, in quanto sepolcro per il corpo del santo. Vincenzo Foppa e chi lo accompagnò nel definire il suo programma iconografico non potevano quindi non tenerlo in debita considerazione.

La creazione di un repertorio iconografico per Pietro da Verona si sviluppa contestualmente la diffusione della sua devozione promossa dall'Ordine dopo il suo martirio. La sua canonizzazione avvenuta nel 1253, a un solo anno della morte, fu occasione per un'opera di sensibilizzazione alla sua devozione attraverso tutti gli strumenti possibili. Già nel 1254 il Capitolo generale aveva dato mandato ai priori di diffondere il culto di san Domenico e di san Pietro martire anche con picturce sicché l'immagine diventò il fondamento dal quale sviluppare agiografia e predicazione ${ }^{18}$.

La fresca memoria su avvenimenti avvenuti pochi anni prima fu occasione per delineare un archivio di testimonianze che sarebbero passate al vaglio degli agiografi per configurare una biografia reale ed esemplare del santo. Infatti nel 1255 il Capitolo generale in Sant'Eustorgio stabilì che coloro tra i frati che fossero a conoscenza di miracoli non ancora noti li trasmettessero al priore di Milano. Nel giro di pochi anni si costituì quindi un corpus rilevante sulla vita e miracoli di san Pietro e nel Capitolo di Pisa del 1276 si determinò che tutti i conventi dell'ordine dovessero tenere la biografia ufficiale del santo composta da Tommaso Agni da Lentini ${ }^{19}$. Il repertorio biografico del santo è tracciato altresì nei testi delle legendæe novæe, testi profondamente legati alla predicazione, dedicati alle espressioni della santità contemporanea, e che si sommarono alla risistemazione, diffusione e volgarizzazione anche del patrimonio agiografico preesistente. San Pietro è presente nella Legenda Aurea di Jacopo da Varagine, nelle Vitce fratrum di fra' Gerardo di Frachet oltre che, in forma ampliata, nel testo biografico di Ambrogio Taegio ${ }^{20}$. 

tesi a insegnamenti etici, come risulta evidente ad esempio rileggendo i Sermones de sanctis di Iacopo da Varagine. Riguardo a Pietro da Verona si aggiunse all'elemento esemplare anche la volontà di ricreare un'empatia con il santo, dettata da uno specifico interesse dell'Ordine a promuovere la devozione nei suoi confronti. Come ben evidenziato da Giovanni Paolo Maggioni nel suo attento contributo sul tema del $2016^{21}$, anche Iacopo da Varagine nel volumen breve dei Sermones insiste su questa volontà. Per questo, se la predicazione dispone gli elementi costitutivi della legenda nella logica dell'argomentazione esemplare, a servizio di un discorso lontano da preoccupazioni storiografiche, invece per san Pietro da Verona c'è interesse che vi siano richiami costanti alla realtà tangibili come luoghi e testimoni.

di imago agens dell'ars memorativa ${ }^{22}$, definita efficacemente da Ricœur «icona verbale» ${ }^{23}$. Le storie legate alla figura di san Pietro si concentrano sulle capacità taumaturgiche e sul potere del suo agire contro l'eresia catara. Sia nell'iconografia che nei sermones riguardanti il santo, il tema principale è il martirio mentre tra i miracoli maggiormente raffigurati vi è quello della nube che rappresenta il santo nella sua funzione di predicatore. Nella Leggenda Aurea (LXI) Jacopo da Varagine descrivendo il martirio di san Pietro pone quattro riferimenti esemplari che caratterizzeranno da quel momento il profilo del santo, affermando che nello stesso giorno Pietro si manifestò come annunciatore della fede, martire, profeta e dottore.

Nel Quattrocento diminuisce la presa dell'immagine del santo come oppositore dell'eresia mentre si potenzia quella della vita perfetta; un esempio di questa tendenza si riscontra nei Sermones de sanctis di Vincenzo Ferrer ${ }^{24}$.

18 Pietro è modello di santità per i fedeli ma soprattutto per i frati che sono invitati a seguirne l'esempio di vita nel contempo attiva e contemplativa.

19 L'iconografia di san Pietro da Verona ${ }^{25}$ sembra rimarcare alcuni aspetti ricorrenti nei sermoni che ne esaltano l'esemplarità: Pietro persegue l'imitatio Christi attraverso la sua passione e morte ${ }^{26} \mathrm{ma}$ anche attraverso l'intera sua vita che è rappresentata come quella di vergine, martire, predicatore.

20 Tra gli episodi della vita, il martirio è ampliamente al centro delle vite e della liturgia del santo così come nella sua rappresentazione figurativa. Pur nelle varianti dell'iconografia, gli artisti che lo raffigurano mantengono fedeltà al racconto. Gli elementi ideali che derivano dallo sviluppo della riflessione sull'esemplarità del santo, trovano rispondenza in elementi che si pongono a corredo della narrazione: l'intreccio dell'elemento biografico con rappresentazioni ideali nell'omiletica, nelle biografie e nella iconografia sono poste in parallelo e non frammiste per evidenziare la concretezza fattuale di vita e morte del santo sulla quale poi disporre in parallelo una riflessione esemplare.

\section{L'arca di Giovanni di Balduccio}

21 All'interno di Sant'Eustorgio il primo importante repertorio iconografico riguardante Pietro da Verona è quello relativo all'arca sepolcrale di Giovanni di Balduccio (fig. 1). L'iconografia riprende episodi in vita e morte probabilmente rielaborati sui testi del cronista domenicano milanese Galvano Fiamma ${ }^{27}$ vissuto a cavallo tra XIII e XIV secolo.

Cahiers d'études italiennes, 29 | 2019 

Catalogus sanctorum et gestorum eorum ex diversis et multis voluminibus collectus ${ }^{29}$, volume tra l'altro presente nella biblioteca del convento di Sant'Eustorgio ${ }^{30}$. Con questo episodio Vincenzo Foppa rappresenta un miracolo delle cronache milanesi, nel cui racconto i fedeli di Sant'Eustorgio potevano ricostruire un percorso reale e noto. Infine, nell'episodio del piede risanato, conosciuto anche come miracolo di Narni (fig. 6), l'aspetto di realtà è enfatizzato dall'atteggiamento degli astanti ma anche dalla sapiente ricostruzione dell'ambientazione architettonica che evidenzia la ricerca di una resa prospettica illusionistica ${ }^{31}$. Gli episodi della vita del santo sono quindi ricondotti a un contesto di storia: gli episodi narrati dall'agiografia del santo sono separati da quello 
che compone invece l'apparato retorico che ne evidenzia virtù e carismi. La resa realistica delle scene riconduce a quello che Arasse descrive così:

Per lo spettatore umanista almeno credere all'illusione dell'immagine poggia su una convenzione tacita: deve accettare di 'Giocare al gioco' dell'illusione. Così che la credibilità dell'immagine, l'illusione che ella suscita tiene insieme una doppia retorica: quella dell'immagine - che persuade della sua verità, della probabilità della sua esistenza- e quella del discorso tenuto sull'illusione prestata all'immagine. Il valore propriamente artistico della rappresentazione figurata tiene, in questo sistema di convenzioni, alla capacità che ha di suscitare e di sostenere il gioco di queste due retoriche ${ }^{32}$.

Le considerazioni riconducibili all'interpretazione della santità di Pietro sono invece sviluppate negli altri elementi del ciclo: le scene mariane di Annunciazione e Assunzione (figg. 7 e 8), i santi e la superficie a scaglie policrome della cupola.

Le scene mariane trovano spazio sullo stesso registro delle storie di san Pietro, e mantengono con quelle una corrispondenza di dimensioni tra le figure che le animano e gli ambienti rappresentati. La compresenza parallela delle due narrazioni non può essere semplicisticamente liquidata come un attestato di devozione al santo martire domenicano e alla Vergine. Come sottolineato precedentemente, la cappella si proponeva lo scopo precipuo di conservare la testa di san Pietro e, pertanto, anche la narrazione per immagini risulta corrispondere alla devozione verso il santo. Le chiavi di lettura possono essere riconducibili a diversi fattori, non escludendo che le scelte iconografiche intendessero assommarne i significati. Sinteticamente le possiamo riferire a tre principali. La prima riguarda l'operato di san Pietro presso Sant'Eustorgio dove, tra l'altro promosse, o almeno ispirò, la fondazione di due confraternite: una fu la Societas fidelium, con incarichi relativi all'Inquisizione, fondata con l'obiettivo di coinvolgere anche devoti laici nella lotta contro le eresie e formare un braccio secolare all'azione dell'Inquisizione. La congregazione ebbe molto successo, tanto che l'operato della popolazione milanese contro l'eresia fu lodata in una lettera di Gregorio IX all'arcivescovo di Milano datata primo dicembre $1232^{33}$. Dopo il martirio di Pietro da Verona, la congregazione assunse il nome di Societas sancti Petri Martiris.

La seconda congregazione era invece dedicata alla Vergine con la finalità di promuovere la devozione alla Vergine.

30 Le storie della Vergine insieme a quelle di san Pietro potrebbero quindi essere una rappresentazione delle due anime delle confraternite di Sant'Eustorgio che in questo modo vengono entrambe valorizzate.

31 La seconda chiave di lettura porrebbe al centro la riflessione teologica e di missione dei Domenicani: l'Annunciazione richiamerebbe la divina maternità di Maria che i Catari negavano, perché negavano la realtà di Cristo, Dio e uomo; sostenendo che Cristo era un angelo mandato da Dio ad insegnare la via della perfezione, l'Assunzione potrebbe essere un riferimento alla cosiddetta Pentecoste domenicana, in memoria del 15 agosto 1217 quando Domenico inviò i suoi frati a predicare nel mondo. Maria per i Domenicani non è un oggetto di vaga devozione ma esempio come Sedes Sapientice.

La terza chiave di lettura è maggiormente di tipo narrativo e porrebbe in evidenza la vita di san Pietro come imitazione di Cristo, racchiusa tra il momento dell'annuncio e l'assunzione di Maria, secondo la tradizione già descritta e legata ad agiografia e predicazione. Questo è certamente un punto centrale e rimarca il costante richiamo a questo parallelo a partire dal testo della bolla di canonizzazione e ribadito da Iacopo da Varagine e Tommaso Agni ${ }^{34}$. Ogni episodio presenta alcuni dettagli che potrebbero 
essere interpretati alla luce della letteratura domenicana. Un particolare suggestivo evidenziato dalla Scotti ${ }^{35}$ riguarda un'attenta lettura della scena dell'Assunzione della Vergine, considerata in riferimento agli scritti di sant'Antonino da Firenze. Antonino si sofferma sulla definizione di Maria come Stella maris, proponendo l'immagine del mare come simbolo dell'esistenza e dove l'umanità peccatrice è naufraga. Antonino riprende il parallelo marittimo indicando come possibili elementi per la salvezza tre navi che rappresentano la Chiesa, la Religione, e la Penitenza ${ }^{36}$. Nell'affresco di Foppa è rappresentato un ambiente marino solcato da tre navi su uno sfondo dove spicca una collina con un castello turrito che pare evocare la fortezza che negli scritti di Antonino è usata come figura della vita religiosa.

Da questo spunto si può allargare lo sguardo sugli altri elementi del ciclo pittorico, infatti anche la presenza delle raffigurazioni dei dottori della Chiesa richiama la terza virtù che caratterizza la figura di Pietro nella rappresentazione attraverso la parola a partire dalla bolla di canonizzazione e simboleggiata dalle cosiddette tre aureole o tre corone: di martire, di vergine, di dottore.

Infine, anche il tema della cupola adorna di scaglie policrome (fig. 9) non è estraneo all'azione narrativa complessiva, rappresentando una dimensione escatologica che negli studi domenicani è presente e introduce alla dimensione simbolica della raffigurazione dell'inimmaginabile secondo ragione ${ }^{37}$. In un impianto iconografico così regolato, anche le figure di santi che si affacciano nei tondi alternati ai finestroni nell'imposta della cupola dovevano rientrare nella narrazione complessiva. La mancanza di attributi che ne permetta un chiaro riconoscimento non ci consente ipotesi fondate.

Per concludere, è necessario considerare che la perdita di parte dell'apparato decorativo della cappella, come ad esempio la cupola del vano dove è collocato l'altare, non ci consente di delineare con certezza il programma iconografico complessivo della cappella quindi di individuare un eventuale, preciso riferimento a uno o più testi di riferimento; tuttavia gli elementi presenti definiscono chiaramente una struttura compositiva narrante che riproduce la specificità della promozione del culto di san Pietro martire. Non si può ridurre l'apporto dei Domenicani nelle scelte iconografiche del Foppa a richiedere immagini ben comprensibili e con intento didascalico. Il ciclo infatti segue una speciale cura di rimandi e collegamenti, in un racconto con elementi tratti dal repertorio di immagini per parole diffuse attraverso la predicazione e letteratura domenicane. Solo una lettura del ciclo che tenga in dovuto conto gli intrecci tra espressioni figurative e letterarie consente un'interpretazione maggiormente conforme al significato originale dell'opera. 
Fig. 1. - Giovanni di Balduccio, Arca di San Pietro Martire, 1335-1339, marmo di Carrara e marmo rosso di Verona scolpiti, Cappella Portinari in Sant'Eustorgio.

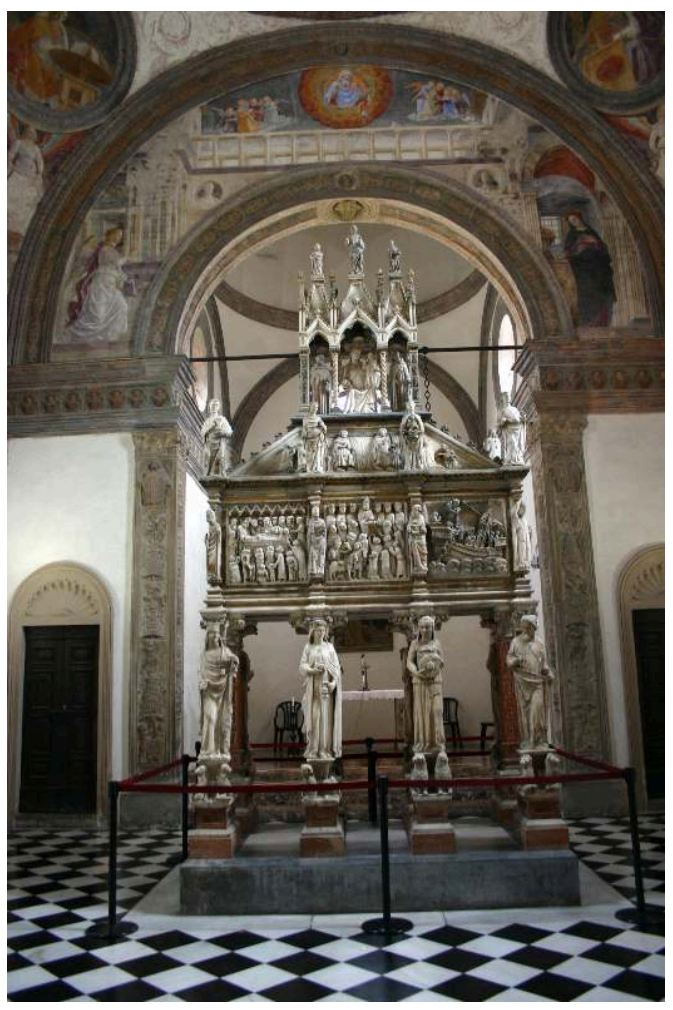

Foto di Giovanni Dall'Orto.

Fig. 2. - Giovanni di Balduccio, dettaglio dell'arca di San Pietro Martire con episodio del miracolo del salvataggio della nave nel mare in tempesta e testa della Prudenza, marmo di Carrara scolpito.

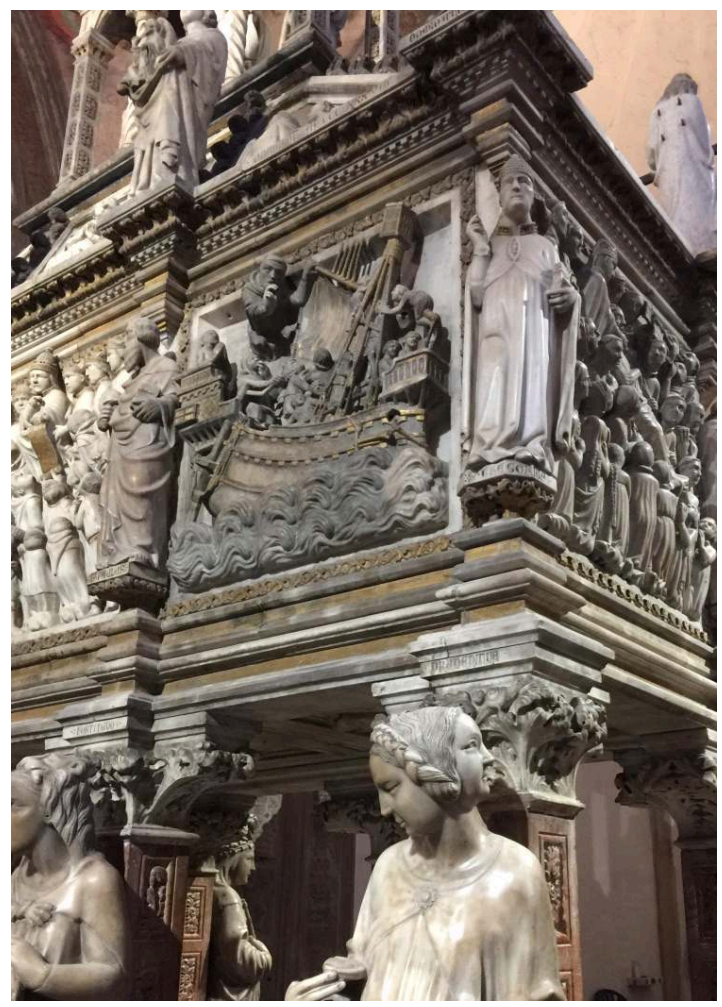


Fig. 3. - Vincenzo Foppa, Martirio di san Pietro da Verona, affresco, Cappella Portinari in Sant'Eustorgio, arcone a sinistra.

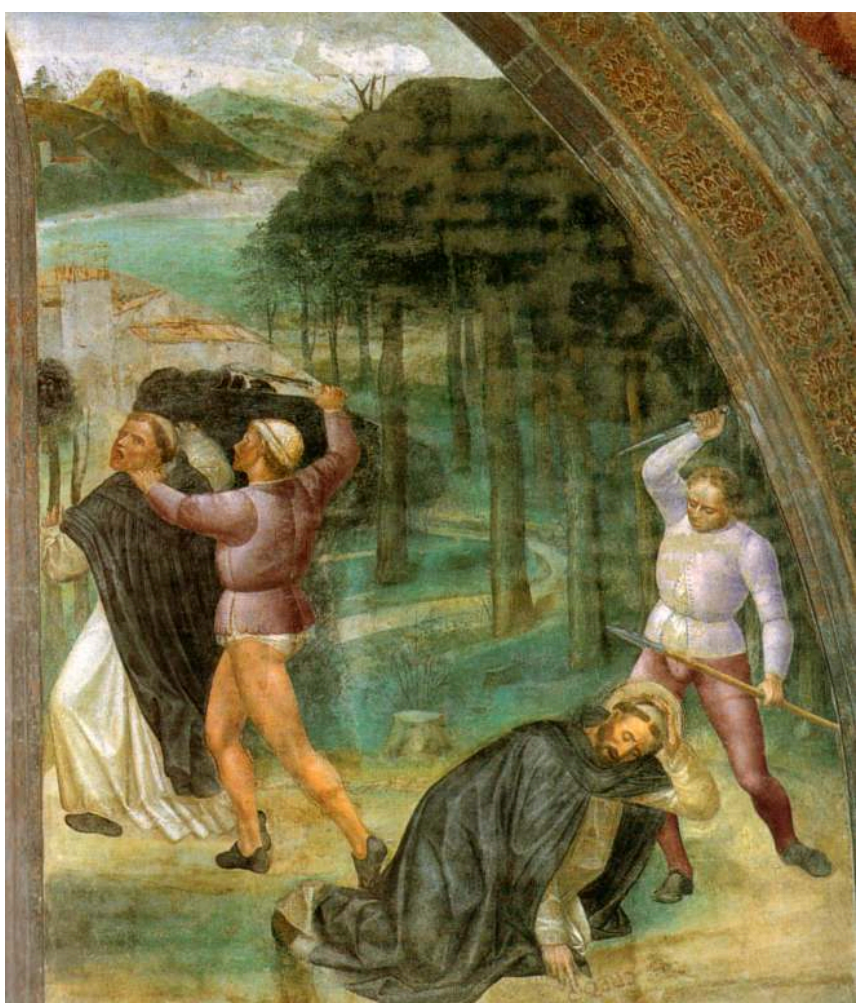

Fig. 4. - Vincenzo Foppa, Il miracolo della nube e disvelamento della falsa Madonna, affresco, Cappella Portinari in Sant'Eustorgio, arcone a destra.

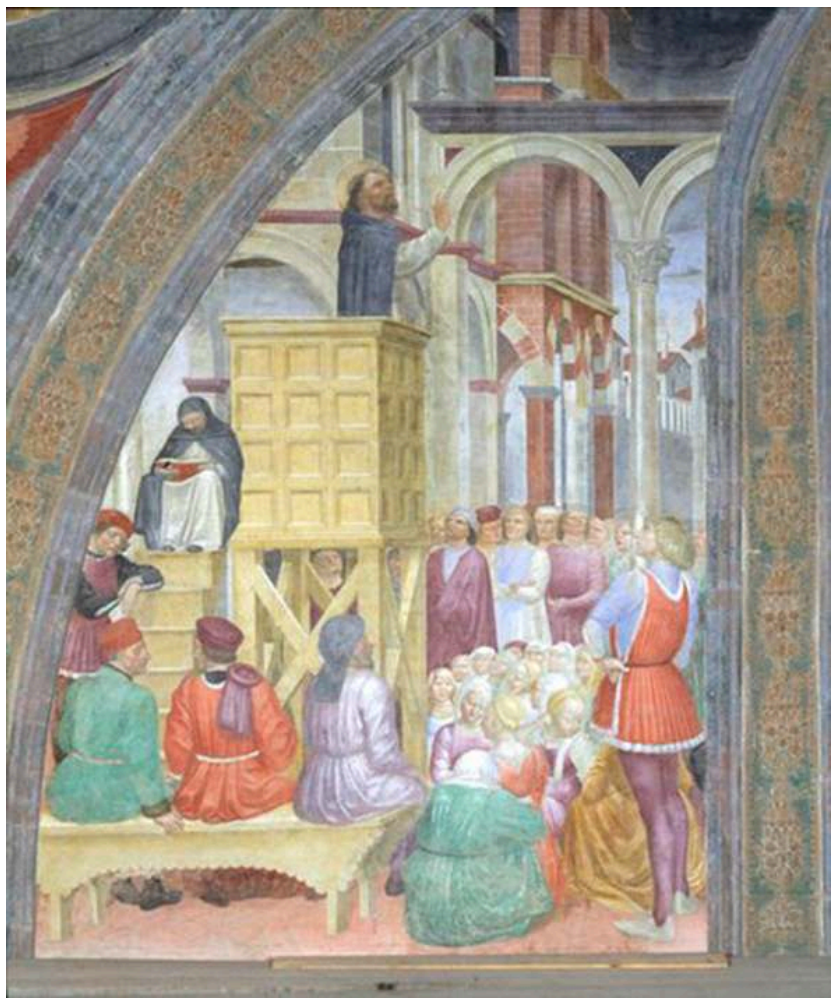


Fig. 5. - Vincenzo Foppa, Disvelamento della falsa Madonna, affresco, Cappella Portinari in Sant'Eustorgio, arcone a destra.

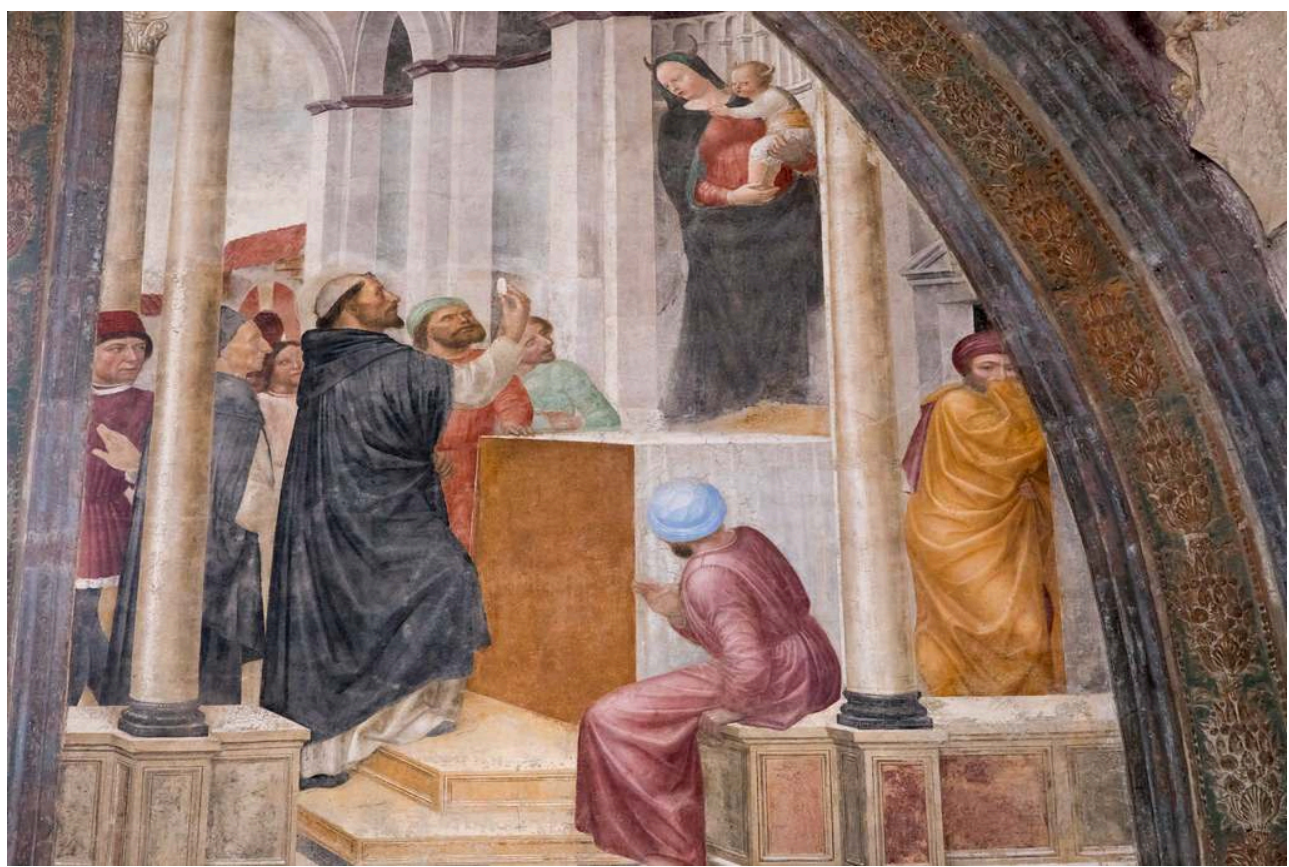

Fig. 6. - Vincenzo Foppa, II miracolo del piede risanato, affresco, Cappella Portinari in Sant'Eustorgio, arcone a sinistra.

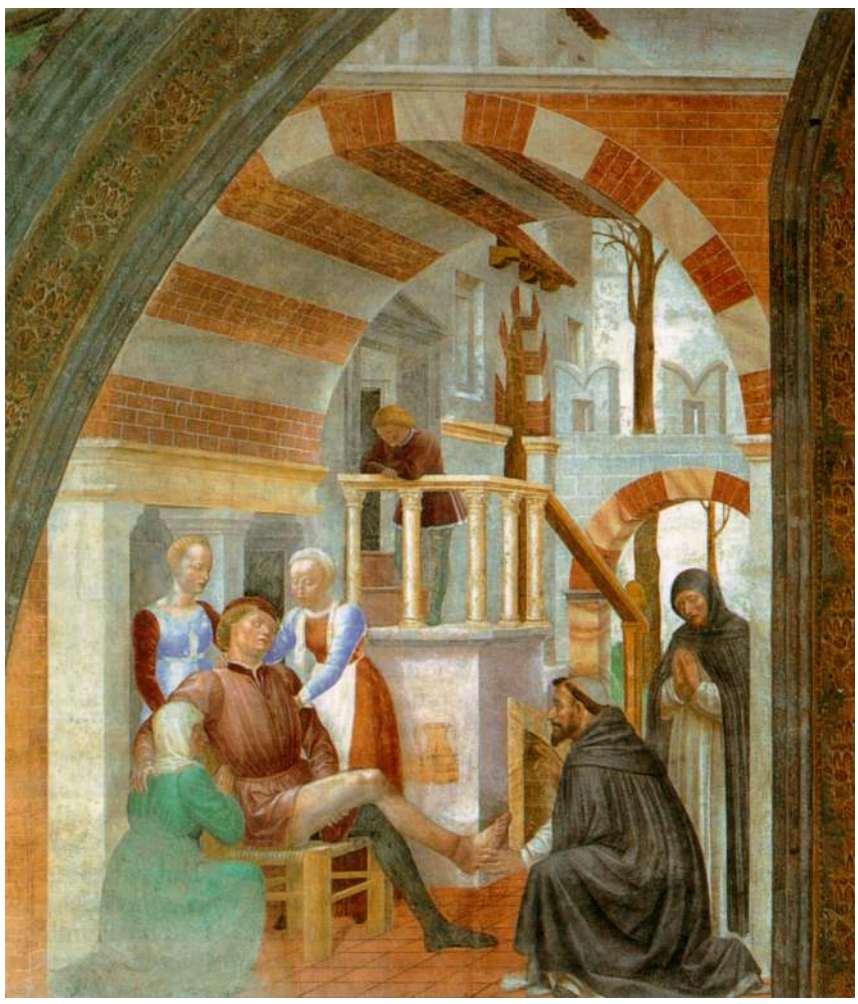


Fig. 7. - Vincenzo Foppa, Annunciazione, affesco, arcone su parete di fondo.

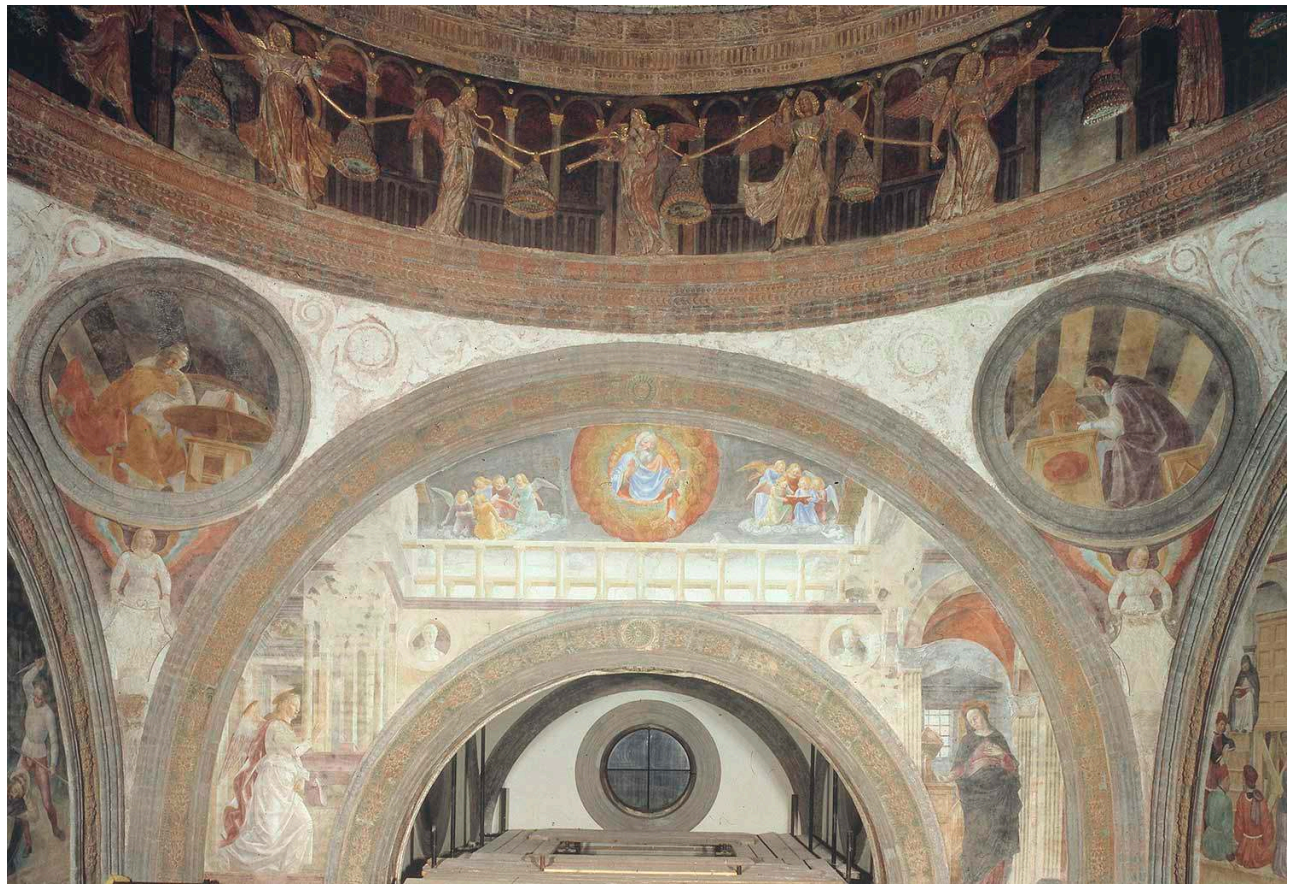

Fig. 8. - Vincenzo Foppa, Assunzione, affresco, arcone su parete d'ingresso.

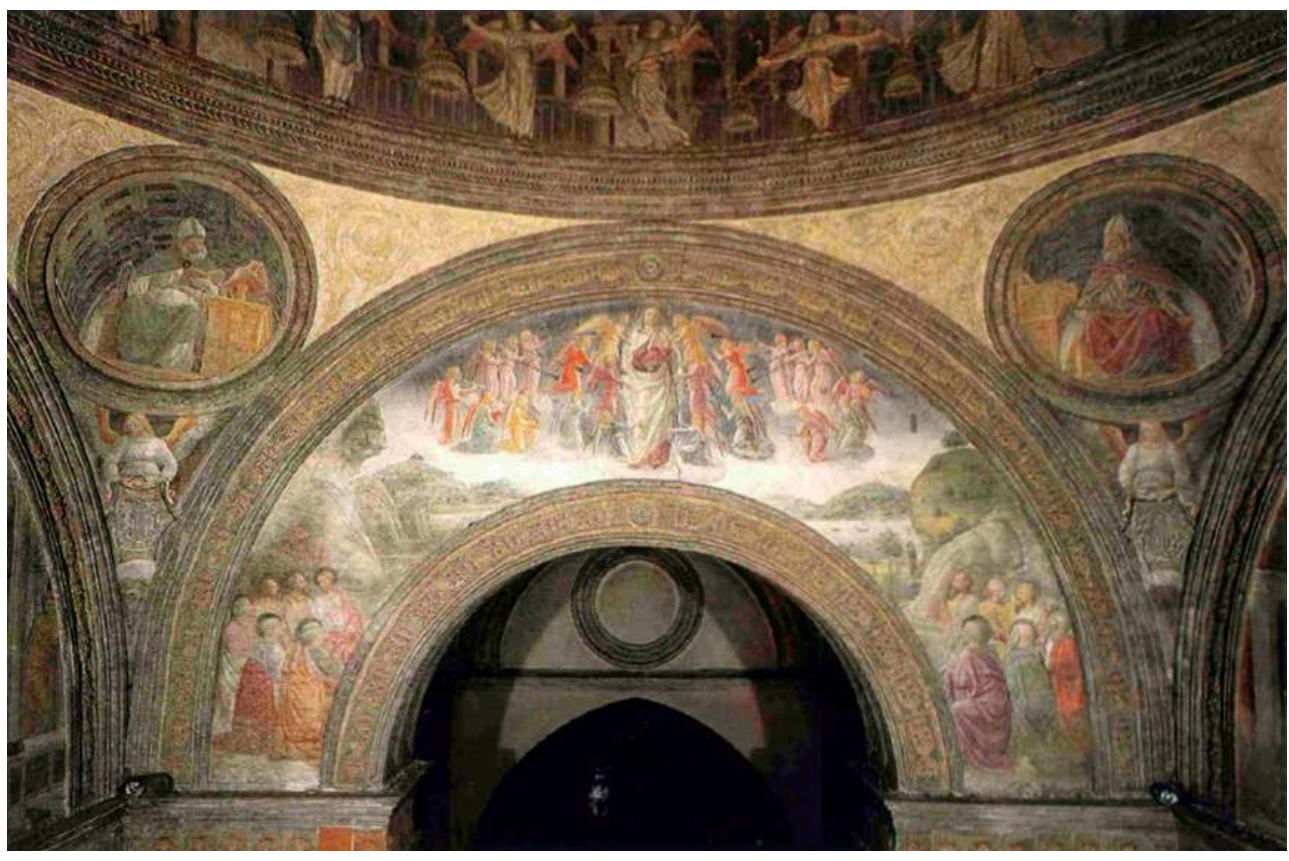


Fig. 9. - Vincenzo Foppa, cupola a scaglie policrome, affresco, Cappella Portinari in Sant'Eustorgio, cupola.

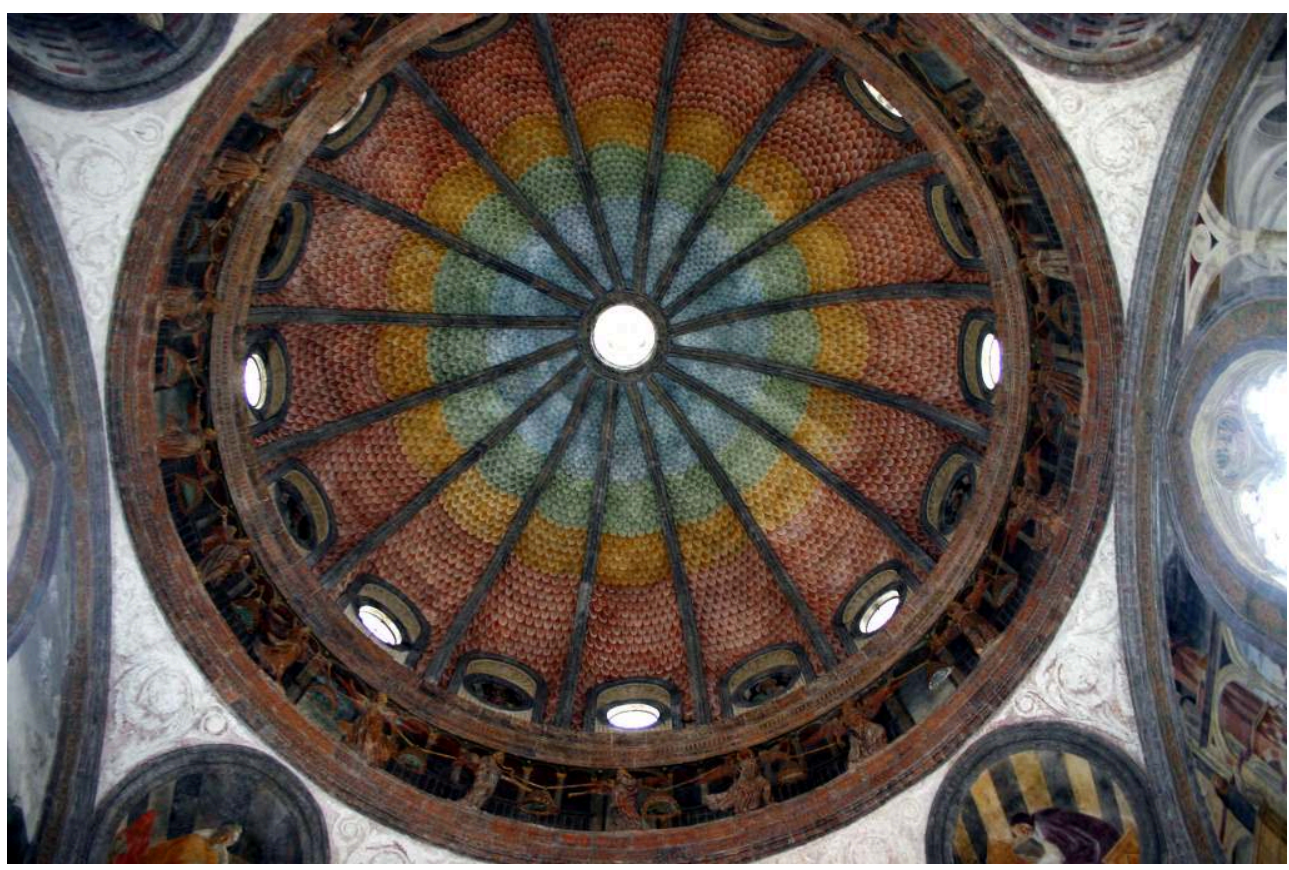

Fig. 10. - Vincenzo Foppa, dettaglio dell'arcone della parete di ingresso, in basso a destra.

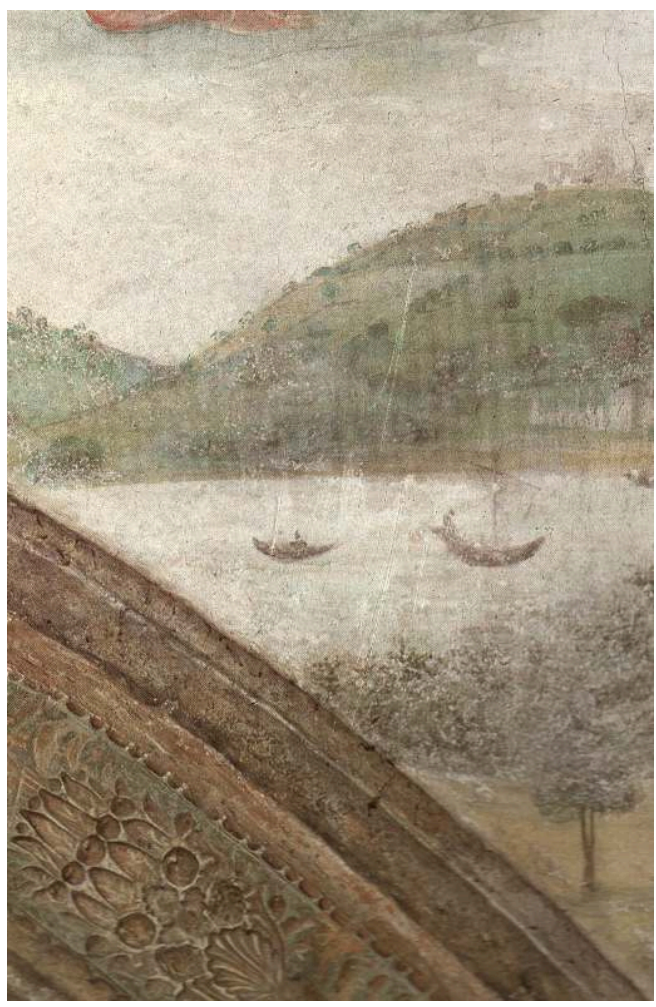




\section{BIBLIOGRAFIA}

ALCE Venturino o. p., Iconografia di san Pietro da Verona martire domenicano, «Memorie Domenicane», vol. LXIX, 1952, pp. 100-114 e 150-168.

ALCE Venturino o. p., L'iconografia di san Pietro Martire nel settimo centenario del suo martirio, «Arte Cristiana», vol. XL, fasc. 39, 1952, pp. 166-171.

ALCE Venturino o. p., La tomba di san Pietro martire e la cappella Portinari in Sant'Eustorgio di Milano, «Memorie Domenicane», vol. LXIX, fasc. I, 1952, p. 3-34.

AllegranZa Giuseppe, Descrizione della basilica di S. Eustorgio, 1784, ms., Milano, S. Eustorgio, Archivio Parrocchiale.

ARASSE Daniel, Entre dévotion et culture : fonctions de l'image religieuse au $X V^{e}$ siècle, in Faire croire. Modalités de la diffusion et de la réception des messages religieux du XII ${ }^{e} a u \mathrm{XV}^{e}$ siècle, Actes de la table ronde de Rome (22-23 giugno 1979), n 51, Roma, École française de Rome, 1981, p. 131-146.

ARSLAN Edoardo, La scultura nella seconda metà del Quattrocento, in Storia di Milano, vol. VII, Milano, Fondazione Treccani degli Alfieri per la storia di Milano, 1956, pp. 713-724.

BARONI Costantino, Scultura gotica-lombarda, Milano, Edizioni d'arte Emlio Bestetti, 1944.

BENEDETTI Marina, Inquisitori lombardi del Duecento, Roma, Edizioni di Storia e Letteratura, 2008.

BugatTI Girolamo, Storia del convento di S. Eustorgio, ms., ASM, Fondo Religione, p.a., 1103, 1577.

CATTANEo Enrico, Le vicende storiche, in G. A. Dell'Acqua (a cura di), La basilica di Sant'Eustorgio in Milano, Milano, Banca Popolare, 1984, pp. 17-43.

Dalai EMILIANI Marisa, Per la prospettiva «padana»: Foppa rivisitato, «Arte Lombarda», vol. XVI, 1971, pp. 117-136.

Dalai Emiliani Marisa, Il ciclo del Foppa nella cappella Portinari, in G. A. Dell'Acqua (a cura di), La basilica di Sant'Eustorgio in Milano, Milano, Banca Popolare, 1984, pp. 155-171.

DA VARAZZE Iacopo, Legenda Aurea, testo critico riveduto e commentato a cura di G. P. Maggioni, Firenze, SISMEL Edizioni del Galluzzo, 2007.

DE FRACHETO Gerardus, Vitce fratrum Ordinis Prcedicatorum necnon cronica ordinis ab anno 1203 usque ad 1254: volumen unicum, in B. M. Reichert (a cura di), Monumenta Ordinis Fratrum Prcedicatorum Historica, vol. I, Louvain-Roma-Paris, Istituto Storico Domenicano, 1896.

DELCORNo Carlo, Exemplum e letteratura tra Medioevo e Rinascimento, Bologna, il Mulino, 1989.

DonDAINE Antoine, Saint Pierre Martyr. Études, «Archivum fratrum prædicatorum», vol. XXIII, 1953, pp. 66-162.

FASOLI Sara, Perseveranti nella regolare osservanza, I predicatori osservanti nel ducato di Milano (secc. XV-XVI), Milano, Edizioni Biblioteca Francescana (coll. Studi di Storia del Cristianesimo e delle Chiese cristiane, vol. XVI), 2011.

FESTA Gianni (a cura di), Martire per la fede. San Pietro da Verona domenicano e inquisitore, Bologna, ESD, 2007. 
Fratris Galuagni De la Flamma, Chronica Ordinis praedicatorum ab anno 1170 usque ad 1333, in B. M. Reichert (a cura di), Monumenta Ordinis Fratrum Prcedicatorum Historica, vol. II, fasc. 1, RomæStuttgardiæ, 1897.

FumAGalli Edoardo, Francesco Sforza e i domenicani Gioacchino Castiglioni e Girolamo Visconti, «Archivium Fratrum Predicatorum», vol. LVII, 1987, pp. 45-101.

GIORDANO Luisa, Prima degli affreschi: la struttura architettonica, in L. Mattioli Rossi (a cura di), Vincenzo Foppa. La cappella Portinari, Milano, Federico Motta editore, 1999, pp. 17-34.

GitLIN BERNSTEIN Jo Anne, Science and Eschatology in the Portinari Chapel, «Arte lombarda», $\mathrm{n}^{\circ}$ 60, 1981, p. 33-40.

KAEPPELI Thomas, La bibliothèque de Saint Eustorge à Milan à la fin du XVe siècle, "Archivium Fratrum Prædicatorum», vol. XXV, 1955, pp. 5-74.

KAFTAL George, Saints in Italian Art, 4 voll., Firenze, Sansoni, 1952-1985.

Prosperi Adriano, LAVEnia Vincenzo e TEDeschi John (a cura di), Pietro Martire, santo, in Dizionario storico dell'Inquisizione, vol. III, Pisa, Scuola Normale Superiore, 2010, pp. 1209-1210.

MAGGIONI Giovanni Paolo, La figura di Pietro Martire nelle raccolte di sermoni ‘De Sanctis' di Iacopo da Varagine. Considerazioni e testi, in E. Lombardo (a cura di), Models of Virtues. The Roles of Virtues in Sermons and Hagiography for New Saints' Cult (13th to 15th Century), Padova, Centro Studi Antoniani, 2016, pp. 91-120.

RicœUR Paul, La métaphore vive, Paris, Seuil, 1975.

Russoli Franco, Arte lombarda dai Visconti agli Sforza, Milano, Comune di Milano, 1958.

Russoli Franco, Le sculture, in R. Cipriani, G. A. Dell'Acqua e F. Russoli (a cura di), La cappella

Portinari in Sant'Eustorgio a Milano, Milano, Banca Popolare di Milano, 1963.

Scотті Giuseppina, Alcune ipotesi di lettura per gli affreschi della Cappella Portinari alla luce degli scritti di S. Antonino vescovo di Firenze, «Arte Lombarda», $n^{\circ}$ 64, 1983, pp. 65-78.

VICAIRE Humbert, Storia di San Domenico, Milano, Paoline Edizioni, 1983.

VIGEZZI Silvio, Catalogo descrittivo ragionato e critico delle sculture esistenti nella basilica di Sant'Eustorgio in Milano, «Archivio Storico Lombardo», vol. LX, 1933.

WitTGENS Fernanda, Vincenzo Foppa, Milano, Edizioni d'arte Amilcare Pizzi, 1949.

\section{NOTE}

1. Tra gli studi sulla figura di san Pietro da Verona si vedano: A. Dondaine, Saint Pierre Martyr. Études, «Archivum fratrum Prædicatorum», vol. XXIII, 1953, pp.66-162; G. Festa (a cura di), Martire per la fede. San Pietro da Verona domenicano e inquisitore, Bologna, ESD, 2007; M. Benedetti, Inquisitori lombardi del Duecento, Roma, Edizioni di Storia e Letteratura, 2008; A. Prosperi, V. Lavenia e J. Tedeschi (a cura di), Pietro Martire, santo, in Dizionario storico dell'Inquisizione, vol. III, Pisa, Scuola Normale Superiore, 2010, pp. 1209-1210.

2. Pietro fu inquisitore e combatté contro l'eresia catara; il suo martirio avvenne presso un bosco a Barlassina mentre rientrava a Milano da Como. Importante sottolineare che fu anche il primo martire domenicano, il secondo fu San Giovanni di Colonia, vissuto nel XVI secolo e canonizzato solo nel 1867. 
3. E. Cattaneo, Le vicende storiche, in G. A. Dell'Acqua (a cura di), La basilica di Sant'Eustorgio in Milano, Milano, Banca Popolare, 1984, p. 30.

4. E. Fumagalli, Francesco Sforza e i domenicani Gioacchino Castiglioni e Girolamo Visconti, «Archivium Fratrum Prædicatorum», vol. LVII, 1987, pp. 45-101.

5. S. Fasoli, Perseveranti nella regolare osservanza. I predicatori osservanti nel ducato di Milano (secc. XVXVI), Milano, Edizioni Biblioteca Francescana (coll. Studi di Storia del Cristianesimo e delle Chiese cristiane, vol. XVI), 2011, p. 34.

6. H. Vicaire, Storia di San Domenico, Milano, Paoline Edizioni, 1983, p. 494.

7. E. Cattaneo, art. cit., p. 30.

8. S. Fasoli, op. cit., p. 87. Interessante osservare che tra i suoi esecutori testamentari c'era anche Pigello Portinari.

9. La raccolta fondi fu presentata al Capitolo generale dell'Ordine a Londra nel 1335 (le vicende legate alle decisioni della committenza sono esposte in: F. Russoli, Le sculture, in R. Cipriani, G. A. Dell'Acqua e F. Russoli (a cura di), La cappella Portinari in Sant'Eustorgio a Milano, Milano, Banca Popolare di Milano, 1963, p. 31).

10. Il monumento funebre era collocato nella quinta campata sinistra (confronta l'ampia bibliografia storica citata in S. Vigezzi, Catalogo descrittivo ragionato e critico delle sculture esistenti nella basilica di Sant'Eustorgio in Milano, «Archivio Storico Lombardo», vol. LX, 1933) circondata da un recinto di pilastrini in marmo rosso commissionata da Filippo Maria Visconti. Nel 1736 avvenne il primo trasferimento nella cappella Portinari, nel piccolo coro, quindi, nel 1835, fu posizionato al centro della cappella dove si trova tuttora.

11. S. Latuada, Descrizione di Milano, vol. III, Milano, Giuseppe Cairoli, 1737, p. 216 citato in M. Dalai Emiliani, Il ciclo del Foppa nella cappella Portinari, in G. A. Dell'Acqua (a cura di), La basilica, cit., p. 164 , nota 3.

12. G. Bugatti, Storia del convento di S. Eustorgio, ms., ASM, Fondo Religione, p.a., 1103, 1577. Anche G. Allegranza, Descrizione della basilica di S.Eustorgio, 1784, ms., Milano, S. Eustorgio, Archivio Parrocchiale ribadisce la centralità della destinazione della cappella a ospitare il capo di s. Pietro Martire, piuttosto che evidenziare l'obiettivo funerario per Pigello Portinari stesso.

13. L'architetto è ignoto ma gli studi più recenti lo identificano con Guiniforte Solari. Un contributo fondamentale sugli aspetti architettonici della cappella è L. Giordano, Prima degli affreschi: la struttura architettonica, in L. Mattioli Rossi (a cura di), Vincenzo Foppa. La cappella Portinari, Milano, Federico Motta editore, 1999, pp. 17-34.

14. Si ricordano in particolare i fondamentali contributi della Wittgens (F. Wittgens, Vincenzo Foppa, Milano, Edizioni d'arte Amilcare Pizzi, 1949) e della Cipriani (R. Cipriani, G. A. Dell'Acqua e F. Russoli, La cappella Portinari, cit., pp. 111-129).

15. La rilevanza del committente fiorentino è sottolineata in tutti i contributi dedicati all'aspetto architettonico della cappella, talvolta è stata chiamata in causa anche per definire le scelte iconografiche, interpretate come dettate da una vocazione della cappella come preminentemente funeraria come in G. Scotti, Alcune ipotesi di lettura per gli affreschi della Cappella Portinari alla luce degli scritti di S. Antonino vescovo di Firenze, «Arte Lombarda», n 64, 1983, pp. 65-78.

16. M. Dalai Emiliani, Il ciclo del Foppa, art. cit., pp. 161-162.

17. L'aspetto didascalico della narrazione per immagini delle vite dei santi è spesso considerato elemento secondario rispetto alle questioni attributive o stilistiche delle opere. Questo determina spesso la considerazione parziale di elementi che per collocazione sono in naturale dialogo. Artisti successivi chiamati ad intervenire su soggetti analoghi nel medesimo luogo non potevano non tenere conto di quanto già esisteva e la rielaborazione iconografica testimonia una sensibilità legata allo stesso soggetto ma mediata dalla cultura del proprio tempo.

18. Per la diffusione del culto di san Pietro da Verona riferimenti puntuali sono in G. Festa (a cura di), Martire per la fede, cit. 
19. Contributo fondamentale sulle caratteristiche della letteratura esemplare è C. Delcorno, Exemplum e letteratura tra Medioevo e Rinascimento, Bologna, il Mulino, 1989.

20. La Legenda aurea fu composta tra il 1252 e il 1265 dal domenicano Jacopo da Varazze, o da Varagine, entrato nell'ordine nel 1244 e arcivescovo di Genova tra il 1292 e il 1298; il testo si può definire un santorale organizzato secondo l'anno liturgico, contenente 153 adbreviationes di vite dei santi e 23 capitoli dedicati alle principali feste della chiesa cristiana. La sua diffusione, in copie, traduzioni e rielaborazioni, fu enorme. Qui, l'edizione consultata è I. Da Varazze, Legenda Aurea, testo critico riveduto e commentato a cura di G. P. Maggioni, Firenze, SISMEL Edizioni del Galluzzo, 2007, pp.474-497; le Vitce di Gerardo di Frachet sono state pubblicate alla fine del XIX secolo come: Gerardus De Fracheto, Vite fratrum Ordinis Prcedicatorum necnon cronica ordinis ab anno 1203 usque ad 1254: volumen unicum, in B. M. Reichert (a cura di), Monumenta Ordinis Fratrum Prcedicatorum Historica, Louvain-Roma-Paris, Istituto Storico Domenicano, 1896; infine, Ambrogio Taegio rielaborò la Vita s. Petri Martiris di Tommaso Agni che non è giunta a noi nella forma originale. Il testo di Taegio è pubblicato in Acta SS. Aprilis, t. III, Parigi e Roma, 1866, pp. 686-727.

21. G. P. Maggioni, La figura di Pietro Martire nelle raccolte di sermoni 'De Sanctis' di Iacopo da Varagine. Considerazioni e testi, in E. Lombardo (a cura di), Models of Virtues. The Roles of Virtues in Sermons and Hagiography for New Saints' Cult (13th to 15th Century), Padova, Centro Studi Antoniani, 2016, pp. 91-120.

22. C. Delcorno, op. cit., pp. 9-11.

23. P. Ricœur, La métaphore vive, Paris, Seuil, 1975, pp. 265-266.

24. C. Delcorno, op. cit., p. 38.

25. Tra i principali studi sull'iconografia del santo domenicano: V. Alce, Iconografia di san Pietro da Verona martire domenicano, «Memorie Domenicane», vol. LXIX, 1952, pp. 100-114 e 150-168; Id., L'iconografia di san Pietro Martire nel settimo centenario del suo martirio, «Arte Cristiana», vol. XL, fasc. 39, 1952, pp.166-171; G. Kaftal, Saints in Italian Art, 4 voll., Firenze, Sansoni, 1952-1985, pp. 817-833.

26. Iacopo da Varagine, Sermones de sanctis volumen breve, n. 430.

27. La cronaca di Galvano Fiamma è stata pubblicata alla fine del XIX secolo come: Fratris Galuagni De La Flamma, Chronica Ordinis proedicatorum ab anno 1170 usque ad 1333, in B. M. Reichert (a cura di), Monumenta Ordinis Fratrum Prcedicatorum Historica, vol. II, fasc. 1, Romæ-Stuttgardiæ, 1897.

28. Ad esempio: C. Baroni, Scultura gotica-lombarda, Milano, Edizioni d'arte Emlio Bestetti, 1944; E. Arslan, La scultura nella seconda metà del Quattrocento, in Storia di Milano, vol. VII, Milano, Fondazione Treccani degli Alfieri per la storia di Milano, 1956, pp. 699 e sgg; F. Russoli, Arte lombarda dai Visconti agli Sforza, Milano, Comune di Milano, 1958, pp. 8-9. Un testo approfondito sugli aspetti iconografici è V.Alce, La tomba di san Pietro martire e la cappella Portinari in Sant'Eustorgio di Milano, estratto da «Memorie Domenicane», vol. LXIX, fasc. I, 1952.

29. A. Dondaine, Saint Pierre Martyr, art. cit., p. 144.

30. T. Kaeppeli, La bibliothèque de Saint Eustorge à Milan à la fin du XVe siècle, «Archivium Fratrum Prædicatorum», vol. XXV, 1955, pp. 23-26.

31. Gli studi sulla resa prospettica delle opere di Foppa partono da M. Dalai Emiliani, Per la prospettiva «padana»: Foppa rivisitato, «Arte Lombarda», vol. XVI, 1971, pp. 117-136.

32. D. Arasse, Entre dévotion et culture: fonctions de l'image religieuse au $\mathrm{XV} v^{e}$ siècle, in Faire croire. Modalités de la diffusion et de la réception des messages religieux $d u X^{e}{ }^{e}$ au XV siècle, Actes de la table ronde de Rome (22-23 giugno 1979), nº 51, Roma, École française de Rome, 1981, p. 138.

33. E. Cattaneo, art. cit., p. 27.

34. La Bolla Magnis et crebris fu emanata il 25 marzo 1253 da papa Innocenzo IV. In essa è ricostruito il profilo del santo attraverso le sue azioni in vita e i miracoli dopo la morte. Il martirio è il momento centrale della vita e caratterizzato da elementi che lo pongono in relazione alla Passione di Cristo. Nella Legenda Aurea e anche nei Sermones (cfr. G. P. Maggioni, op. cit., 
pp. 99-101) Iacopo da Varaggine riprende il parallelismo che è altresì ripercorso nella Vita di Tommaso Agni (cfr. C. Delcorno, op. cit., pp. 34-35).

35. G. Scotti, art. cit., pp. 69 e 74.

36. S. Antoninus, 1740, lib. I, tit. V, cap. III, coll. 407.

37. J. A. Gitlin Bernstein, Science and Eschatology in the Portinari Chapel, «Arte lombarda», $\mathrm{n}^{\circ} 60$, 1981, p. 35.

\section{RIASSUNTI}

L'articolo propone una lettura complessiva delle storie di san Pietro Martire all'interno della basilica di Sant'Eustorgio a Milano. In particolare si considera il complesso decorativo della Cappella Portinari con gli affreschi di Vincenzo Foppa alla luce di considerazioni multidisciplinari. Queste sono soprattutto tese a evidenziare l'apporto della narrazione domenicana che pone scrittura e rappresentazione figurativa in una relazione di mutuo scambio. Come nella parola espressa nell'agiografia, nel canone liturgico dell'Ordine e nella predicazione ricorrono richiami alle immagini, così nella figurazione si traducono schemi compositivi utilizzati nella parola.

L'article propose une lecture globale des récits de saint Pierre de Vérone dans la basilique de Sant'Eustorgio de Milan. Nous examinons en particulier le complexe décoratif de la chapelle Portinari avec les fresques de Vincenzo Foppa à la lumière de considérations multidisciplinaires. Celles-ci visent avant tout à mettre en évidence la contribution du récit dominicain qui place l'écriture et la représentation figurative dans une relation d'échange mutuel. De la même façon qu'on trouve le recours aux images dans la parole exprimée dans l'hagiographie, le canon liturgique de l'Ordre et la prédication, des schémas de composition identiques à ceux qui sont utilisés dans la parole se présentent dans la figuration.

The paper aims to offer an overall interpretation of Saint Peter Martyr's stories inside Saint Eustorgio's church in Milan. In particular, the article will examine the decorative complex of Portinari's Chapel with Vincenzo Foppa's frescoes, in light of the multidisciplinary considerations. The aim is to highlight the contribution of the Dominican's narrative that puts writing and figurative representation in a relation of mutual exchange. As there are constant references to images in the texts of hagiography, specific Dominican liturgy and homiletics, so composition schemes, characteristic of rhetoric principles, are present in the iconography.

\section{INDICE}

Keywords : Milan, basilica of Saint Eustorgio, Saint Peter of Verona, Vincenzo Foppa, Dominican preaching, Dominican iconography

Mots-clés : Milan, Sant'Eustorgio, saint Pierre de Vérone, Vincenzo Foppa, prédication dominicaine, iconographie dominicaine

Parole chiave : Milano, Basilica di Sant'Eustorgio, Pietro da Verona Martire, Vincenzo Foppa, predicazione domenicana, iconografia domenicana 
AUTORE

RITA CAPURRO

Politecnico de Milan.

rita.capurro@polimi.it 